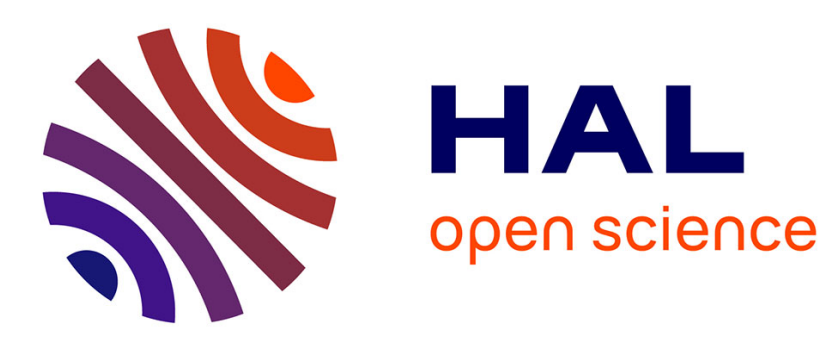

\title{
Effets des dégénérescences Zeeman sur la fluorescence de résonance induite par un laser intense
}

\author{
C. Cohen-Tannoudji, S. Reynaud
}

\section{To cite this version:}

C. Cohen-Tannoudji, S. Reynaud. Effets des dégénérescences Zeeman sur la fluorescence de résonance induite par un laser intense. Journal de Physique Lettres, 1977, 38 (8), pp.173-176. 10.1051/jphyslet:01977003808017300 . jpa-00231353

\section{HAL Id: jpa-00231353 https://hal.science/jpa-00231353}

Submitted on 1 Jan 1977

HAL is a multi-disciplinary open access archive for the deposit and dissemination of scientific research documents, whether they are published or not. The documents may come from teaching and research institutions in France or abroad, or from public or private research centers.
L'archive ouverte pluridisciplinaire HAL, est destinée au dépôt et à la diffusion de documents scientifiques de niveau recherche, publiés ou non, émanant des établissements d'enseignement et de recherche français ou étrangers, des laboratoires publics ou privés. 


\title{
EFFETS DES DÉGÉNÉRESCENCES ZEEMAN SUR LA FLUORESCENCE DE RÉSONANCE INDUITE PAR UN LASER INTENSE
}

\author{
C. COHEN-TANNOUDJI et S. REYNAUD \\ Laboratoire de Spectroscopie Hertzienne \\ Ecole Normale Supérieure et Collège de France, \\ 24, rue Lhomond, 75231 Paris Cedex 05, France
}

(Reçu le 22 février 1977, accepté le 22 mars 1977)

\begin{abstract}
Résumé. - On attire l'attention sur quelques conséquences de la dégénérescence Zeeman des niveaux inférieur et supérieur d'une transition atomique saturée par un laser résonnant intense : on prévoit des spectres de fluorescence complètement différents suivant que la polarisation de détection est parallèle ou perpendiculaire à celle du laser ; les effets de pompage optique pourraient dans certains cas faire disparaître complètement les spectres de fluorescence en régime stationnaire.

Abstract. - Some consequences of the Zeeman degeneracy of the lower and upper states of an atomic transition saturated by an intense resonant laser beam are pointed out ; completely different fluorescence spectra are predicted according as the detection polarization is parallel or perpendicular to the laser one ; in some cases, the steady state fluorescence spectra could disappear as a consequence of optical pumping effects.
\end{abstract}

1. Introduction. - Le but de cette lettre est d'attirer l'attention sur des effets de polarisation qui pourraient être observés dans des expériences de fluorescence de résonance sur un jet atomique irradié à angle droit par un faisceau laser intense.

Jusqu'à présent, les études aussi bien théoriques [1] qu'expérimentales [2] effectuées sur ce problème ont surtout porté sur des atomes à deux niveaux, pour lesquels on peut évidemment oublier le caractère vectoriel du dipôle atomique ainsi que la polarisation de l'onde incidente. Rappelons brièvement les résultats de ces diverses études. Le spectre de fluorescence, monochromatique comme le faisceau laser incident aux faibles intensités (diffusion élastique), se déforme progressivement lorsqu'on augmente l'intensité pour prendre l'allure d'un triplet dont les trois composantes sont (à résonance) situées en $\omega_{\mathrm{L}}, \omega_{\mathrm{L}} \pm \omega_{1}$ ( $\omega_{\mathrm{L}}$ : fréquence du laser, $\omega_{1}$ : fréquence de Rabi caractérisant le couplage atome-laser), les largeurs et poids de ces composantes pouvant être calculés exactement. Les observations expérimentales ont essentiellement porté sur la composante hyperfine $F=2 \rightarrow F=3$ de la transition $\mathrm{D}_{2}$ du sodium. Pour éliminer l'effet de la structure Zeeman (ainsi que celui du pompage hyperfin), on concentre par un pompage optique préalable les atomes dans le sousniveau $F=2, M_{F}=2$ de l'état fondamental, qui ne peut, la polarisation du laser étant $\sigma^{+}$, être couplé qu'au sous-niveau $F=3, M_{F}=3$ de l'état excité, de sorte que l'on est ainsi ramené à un système à deux niveaux.

Nous considérerons ici une situation différente, celle d'une excitation en polarisation $\pi$ d'une transition atomique entre deux niveaux de moments cinétiques non nuls, par exemple la transition $J_{\mathrm{g}}=1 \leftrightarrow J_{\mathrm{e}}=2$ représentée sur la figure 1. Il apparait trois transitions $\pi$ (flèches verticales de la figure 1) dont les amplitudes sont proportionnelles aux coefficients de Clebsch-Gordan indiqués sur la figure. On voit immédiatement que les fréquences de Rabi $\omega_{1}^{1}$ et $\omega_{1}^{0}$ associées respectivement aux transitions $-1 \leftrightarrow-1$ et $+1 \leftrightarrow+1$ d'une part, $0 \leftrightarrow 0$ de l'autre sont différentes (elles sont proportionnelles aux amplitudes des transitions correspondantes). Ainsi que plusieurs auteurs l'ont signalé [3], le spectre de fluorescence $\pi$ doit donc dans ce cas être plus ou

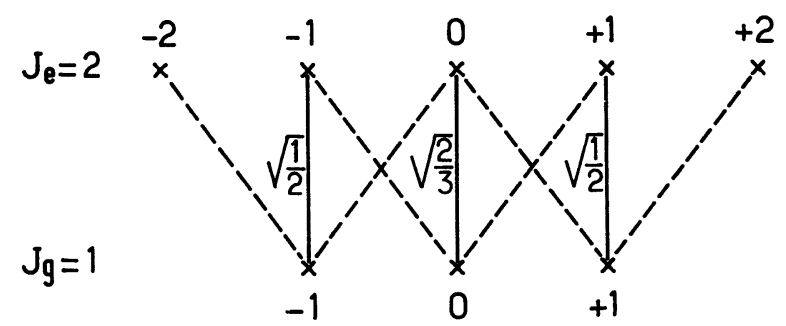

FIG. 1. - Transition atomique $J_{\mathrm{g}}=1 \leftrightarrow J_{\mathrm{e}}=2$; on a indiqué les coefficients de Clebsch-Gordan associés aux transitions $\pi$ verticales.

[Atomic transition $J_{\mathrm{g}}=1$ to $J_{\mathrm{e}}=2$; the Clebsch-Gordan coefficients corresponding to the vertical $\pi$ transitions are indicated.] 
moins une superposition de deux triplets, $\omega_{\mathrm{L}}, \omega_{\mathrm{L}} \pm \omega_{1}^{1}$ et $\omega_{\mathrm{L}}, \omega_{\mathrm{L}} \pm \omega_{1}^{0}$ correspondant à ces deux fréquences de Rabi $\omega_{1}^{1}$ et $\omega_{1}^{0}$. Il ne faut pas oublier cependant que les trois systèmes à deux niveaux $-1 \leftrightarrow-1$, $0 \leftrightarrow 0,+1 \leftrightarrow+1$ de la figure 1 ne sont pas indépendants, mais couplés par l'émission spontanée de photons $\sigma$ (flèches en pointillés de la figure 1), ce qui modifie les largeurs et les poids des diverses composantes et peut, pour certaines valeurs de $J_{\mathrm{e}}$ et $J_{\mathrm{g}}$, faire disparaître complètement le spectre de fluorescence (effets de pompage optique que nous discuterons plus loin).

Une autre possibilité qui ne semble pas avoir été envisagée jusqu'ici serait d'observer le spectre de fluorescence en polarisation $\sigma$ (spectre $\pi-\sigma$ : excitation en $\pi$, observation en $\sigma$ ). A faible intensité, ce spectre est monochromatique (diffusion Raman avec changement de +1 ou -1 du nombre quantique magnétique dans l'état inférieur). Que devient ce spectre à haute intensité et comment se compare-t-il au spectre $\pi-\pi$ (excitation et détection en $\pi$ ) ?

Nous montrons ci-dessous comment une approche théorique introduite récemment [4-5] permet de résoudre simplement ces divers problèmes à la limite des hautes intensités. Nous nous limiterons à une discussion qualitative sans entrer dans le détail des calculs qui ne présentent aucune difficulté de principe. Pour simplifier, nous supposerons qu'aucun champ magnétique statique n'est appliqué et que l'excitation laser est résonnante.

2. Diagramme d'énergie de l'atome habillé. - Les niveaux d'énergie du système atome + mode laser en interaction mutuelle (atome habillé) se groupent en multiplicités $\varepsilon_{n}$ séparées de $\omega_{\mathrm{L}}$. La multiplicité $\varepsilon_{n}$ comprend huit niveaux non perturbés exactement dégénérés :

$$
\left\{|\mathrm{g}, M, n+1\rangle,\left|\mathrm{e}, M^{\prime}, n\right\rangle\right\}
$$

$\left(M^{\prime}=-2, \ldots,+2 ; M=-1, \ldots,+1 ; n:\right.$ nombre de photons). Les deux niveaux $\left|\mathrm{e}, M^{\prime}= \pm 2, n\right\rangle$ qui ne peuvent être peuplés ni par excitation optique, ni par émission spontanée ne jouent aucun rôle et seront ignorés. Les six autres niveaux sont couplés deux à deux par l'interaction $V$ avec le laser, les coefficients de couplage étant proportionnels aux coefficients de Clebsch-Gordan

$$
\langle\mathrm{g}, M, n+1|V| \mathrm{e}, M, n\rangle=\frac{1}{2} \omega_{1}^{M} .
$$

On en déduit les états perturbés de la multiplicité $\varepsilon_{n}$ représentés sur la figure 2 , où

$$
| \pm, M, n\rangle=\frac{1}{\sqrt{2}}(|\mathrm{e}, M, n\rangle \pm|\mathrm{g}, M, n+1\rangle)
$$

a pour énergie : $n \omega_{\mathrm{L}} \pm \frac{1}{2} \omega_{1}^{M}$.

3. Spectres $\pi-\pi$ et $\pi-\sigma .-$ Les fréquences de Bohr de l'atome habillé correspondant à des paires de

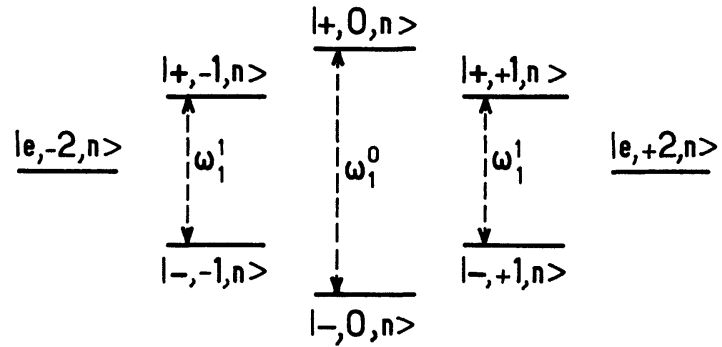

Fig. 2. - Etats perturbés de la multiplicité $\varepsilon_{n}$ de l'atome habillé (les niveaux $|\mathrm{e}, \pm 2, n\rangle$ ne jouent aucun rôle par la suite). La séparation des doublets est donnée par les fréquences de Rabi $\omega_{1}^{1}$ et $\omega_{1}^{0}$. La différence entre $\omega_{1}^{1}$ et $\omega_{1}^{0}$ a été exagérée pour rendre la figure plus claire.

[Perturbed dressed states of the multiplicity $\varepsilon_{n}$ (the levels $|\mathrm{e}, \pm 2, n\rangle$ play no role in the following). The splitting of the doublets is given by the Rabi frequencies $\omega_{1}^{1}$ and $\omega_{1}^{0}$. The difference between $\omega_{1}^{1}$ and $\omega_{1}^{0}$ has been exaggerated to make the figure clearer.]

niveaux entre lesquels le dipôle atomique a un élément de matrice non nul donnent immédiatement les positions des composantes des spectres de fluorescence. Les figures $3 \mathrm{a}$ et $3 \mathrm{~b}$ représentent les transitions permises respectivement en $\pi(\Delta M=0)$ et $\sigma^{-}(\Delta M=-1)$. Ces transitions relient des états appartenant à des multiplicités $\varepsilon_{n}$ et $\varepsilon_{n-1}$ adjacentes.

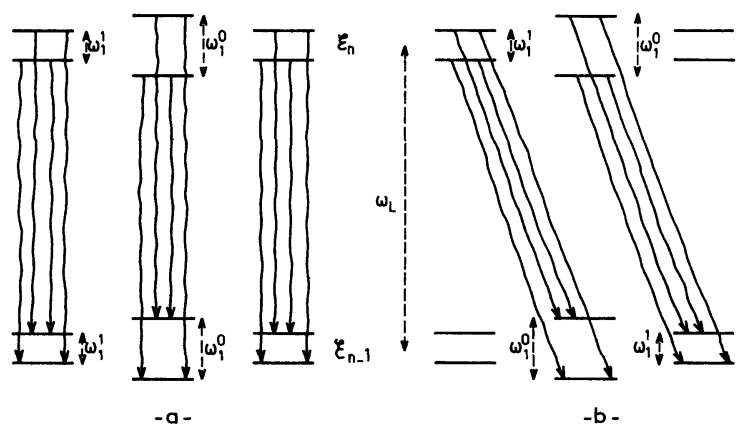

Fig. 3. - Transitions permises, $\pi$ pour la figure $3 a, \sigma^{-}$pour la figure $3 \mathrm{~b}$, apparaissant dans le spectre de fluorescence et reliant les multiplicités $\varepsilon_{n}$ et $\varepsilon_{n-1}$ (la différence entre $\omega_{1}^{1}$ et $\omega_{1}^{0}$ a été exagérée pour rendre la figure plus claire).

[ $\pi$ allowed transitions (Fig. 3a) and $\sigma^{-}$allowed transitions (Fig. 3b) appearing in the fluorescence spectrum and connecting the multiplicities $\varepsilon_{n}$ and $\varepsilon_{n-1}$ (the difference between $\omega_{1}^{1}$ and $\omega_{1}^{0}$ has been exaggerated to make the figure clearer).

On retrouve bien d'abord à partir de la figure $3 \mathrm{a}$, que le spectre $\pi-\pi$ est une superposition de triplets correspondant à des écarts $\omega_{1}^{1}$ et $\omega_{1}^{0}$ différents et dont la composante centrale est toujours située en $\omega_{\mathrm{L}}$. $\mathrm{Si} \omega_{1}^{0}-\omega_{1}^{1}$ est plus grand que la largeur des raies (de l'ordre de la largeur naturelle $\Gamma$ de l'état excité), on obtient cinq raies dont les positions sont représentées sur la figure 4a.

Il apparaît clairement sur la figure $3 b$ que les transitions $\sigma^{-}$(ainsi que $\sigma^{+}$) relient des doublets dont la séparation est différente de sorte que la composante centrale à la fréquence $\omega_{\mathrm{L}}$ disparaît et est remplacée par un doublet $\omega_{\mathrm{L}} \pm \frac{1}{2}\left(\omega_{1}^{0}-\omega_{1}^{1}\right)$; 


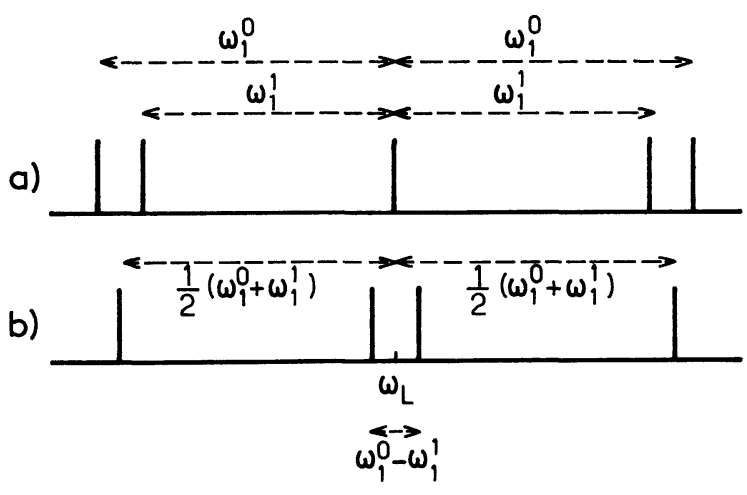

FIG. 4. - Positions des composantes des spectres de fluorescence associées aux transitions des figures $3 \mathrm{a}$ et $3 \mathrm{~b}$. Les spectres $\pi-\pi$ (4a) et $\pi-\sigma(4 b)$ ont une structure différente.

[Positions of the components of the fluorescence spectra associated with the transitions of figures $3 a$ and $3 b$. The $\pi-\pi(4 a)$ and $\pi-\sigma(4 b)$ spectra have a different structure.]

deux composantes latérales apparaissent en $\omega_{\mathrm{L}} \pm \frac{1}{2}\left(\omega_{1}^{0}+\omega_{1}^{1}\right)$. Le spectre $\pi-\sigma$ représenté en $4 \mathrm{~b}$ est donc complètement différent du spectre $\pi-\pi\left({ }^{1}\right)$.

L'approche précédente nous a permis de prévoir très simplement la structure des divers spectres observables lorsqu'on fait varier la polarisation à la détection. Pour calculer de manière précise le poids et la largeur des diverses composantes, il suffirait d'utiliser les résultats établis dans la référence [4] à partir de la résolution de l'équation pilote décrivant l'effet de l'émission spontanée de l'atome habillé. Mentionnons toutefois que certaines raies des spectres des figures 4 correspondent à plusieurs transitions de même fréquence sur la figure 3 et qu'il faut donc dans ce cas tenir compte des couplages par émission spontanée entre les éléments non diagonaux correspondants de la matrice densité (voir appendice de [4] ou appendice B de [5]). Il faut également tenir compte de tels couplages lorsque la séparation $\left(\omega_{1}^{0}-\omega_{1}^{1}\right)$ des doublets des figures 4 n'est plus assez grande devant $\Gamma$ pour que ces doublets soient résolus. Les poids des diverses composantes s'obtiennent en multipliant par des taux de transition les populations stationnaires des niveaux de l'atome habillé calculés en résolvant les équations d'évolution de ces populations. Notons que ces populations stationnaires résultent de l'action combinée de l'excitation optique $\pi$

( $\left.{ }^{1}\right)$ Notons que le cas d'une transition $J_{\mathrm{g}}=\frac{1}{2} \rightarrow J_{\mathrm{e}}=\frac{1}{2}$, qui vient de faire l'objet d'une publication [6], n'est pas très intéressant pour observer ces effets de polarisation puisqu'une seule fréquence de Rabi est associée aux 2 transitions $\pi$ saturées $-\frac{1}{2} \rightarrow-\frac{1}{2}$ et $+\frac{1}{2} \rightarrow+\frac{1}{2}$. Par rapport au triplet de fluorescence d'un système à 2 niveaux, il y a seulement (sur le spectre $\pi-\sigma$ ) une modification des largeurs des raies latérales due au couplage par émission spontanée de photons $\sigma$.

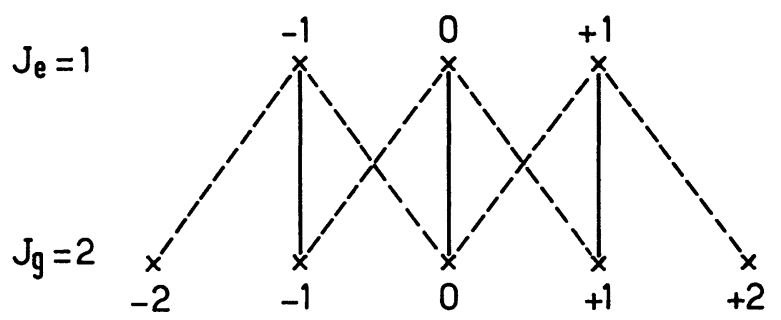

FIG. 5. - Transition atomique $J_{\mathrm{g}}=2 \leftrightarrow J_{\mathrm{e}}=1$. Par pompage optique, les atomes s'accumulent dans $|\mathrm{g}, M= \pm 2\rangle$ et ne peuvent plus absorber de photons laser $\pi$.

[Atomic transition $J_{\mathrm{g}}=2$ to $J_{\mathrm{e}}=1$. Optical pumping accumulates atoms in sublevels $|\mathrm{g}, M= \pm 2\rangle$ from which it is impossible to absorb $\pi$ laser photons.]

qui, étant très intense, égalise les populations de chaque paire de sous-niveaux $|\mathrm{g}, M\rangle$ et $|\mathrm{e}, M\rangle$ et de l'émission spontanée qui redistribue ces populations entre les diverses valeurs de $M$.

Il est absolument indispensable de tenir compte de cet effet de pompage si l'on veut comprendre quantitativement les poids des diverses composantes. Dans certains cas, il peut même être responsable de la disparition des spectres de fluorescence en régime stationnaire. Supposons par exemple que l'on inverse les moments cinétiques de la figure 1 en prenant $J_{\mathrm{g}}=2, J_{\mathrm{e}}=1$ (Fig. 5). L'effet du pompage optique est d'accumuler, après un régime transitoire durant un temps de l'ordre de $\Gamma^{-1}$, tous les atomes dans les états $|\mathrm{g}, M= \pm .2\rangle$ qu'ils ne peuvent plus ensuite quitter par absorption d'un photon laser $\pi$. La fluorescence n'existe alors que pendant le régime transitoire. Il est facile de voir que cette situation se produit chaque fois que $J_{\mathrm{g}}=J_{\mathrm{e}}+1$ ou que $J_{\mathrm{e}}=J_{\mathrm{g}}$ entier ; dans ce dernier cas en effet, le coefficient de Clebsch-Gordan correspondant à la transition $\left|J_{\mathrm{g}}, M=0\right\rangle \leftrightarrow\left|J_{\mathrm{e}}, M=0\right\rangle$ s'annule ce qui interdit aux atomes pompés dans $\left|J_{\mathrm{g}}, M=0\right\rangle$ de s'en échapper par absorption d'un photon laser $\pi$.

En conclusion, on peut dire que la fluorescence de résonance d'un jet atomique irradié par un laser intense ne peut, lorsqu'il y a des dégénérescences Zeeman, s'analyser simplement en termes de superposition de triplets ayant des séparations différentes. On dispose maintenant d'un paramètre supplémentaire qui est l'angle entre les polarisations d'excitation et de détection. L'allure des spectres de fluorescence à haute intensité doit en général changer complètement lorsqu'on fait varier cet angle et il serait intéressant d'essayer d'observer expérimentalement un tel effet. Il faut également se méfier des effets de pompage qui peuvent, dans de nombreux cas, diminuer considérablement l'intensité des spectres en régime stationnaire. 


\section{Bibliographie}

[1] Mollow, B. R., Phys. Rev. 188 (1969) 1969. Une liste détaillée de références plus récentes est donnée dans [4] et [5].

[2] Schuda, F., Stroud, C. R. and Hercher, M., J. Phys. B 7 (1974) L-198 ;

Walther, H., Proc. of the 2nd Laser Spectroscopy Conf., June 75, editors S. Haroche, J. C. Pebay-Peyroula, T. W. Hansch and S. H. Harris (Berlin : Springer Verlag) 1975, 358;

Hartig, W., Rasmussen, W., Schieder, R. et Walther, W., Z. Phys. A 278 (1976) 205;

Wu, F. Y., Grove, R. E. et EzeKIEL, S., Phys. Rev. Lett. 35 (1975) 1426 ;
Grove, R. E., Wu, F. Y. et Ezekiel, S., Phys. Rev. to be published (1977).

[3] Voir [2];

Delsart, C. et Keller, J. C., J. Phys. B 9 (1976) 2769 ;

HigGins, R. B., J. Phys. B 8 (1975) L-321.

[4] Cohen-Tannoudi, C. et Reynaud, S., J. Phys. B 10 (1977) to be published.

[5] Reynaud, S., Thèse de troisième cycle, Paris (1977).

[6] Polder, D. et Schuurmans, M. F. H., Phys. Rev. A 14 (1976) 1468. 